\title{
Underpowered Aircraft -- Performance and Operational Possibilities
}

\author{
Andrew S. Ezzard ${ }^{1}$, Michael R. Vallone ${ }^{2}$, and Robert A. McDonald, Ph.D. ${ }^{3}$ \\ California Polytechnic State University, San Luis Obispo, CA 93407
}

\begin{abstract}
A unique configuration, known as an Underpowered Aircraft, allows for the modification of gliding flight vehicles for increased range and lower cost when compared with fully powered flight vehicles. Intentionally under-sizing the powerplant for a flight vehicle allows the designer to choose a powerplant that will not only perform the mission requirements, but will also provide the customer with the most cost effective solution, as some missions may not require fully powered flight. Specifically, the underpowered aircraft concept studied in this paper is a gliding flight aircraft that does not have enough power for climbing or level flight, but does have enough power to overcome some of the drag forces associated with flight, in turn increasing the effective range of the vehicle. In this paper, the underpowered aircraft concept was analyzed and its feasibility was determined. Analysis done using equations of motion, followed by a more accurate numerical integration including a thrust lapse, determined that the underpowered aircraft concept provides a unique method for a cost effective range extension technology for gliding flight vehicles. Finally, the technology and methods of this paper were applied to the AGM-154 JSOW and JSOW-ER glide munitions and it was determined that JSOW-ER is representative of an underpowered aircraft with our analysis. This paper represents a "back-of-the-envelope" investigation into the underpowered aircraft concept.
\end{abstract}
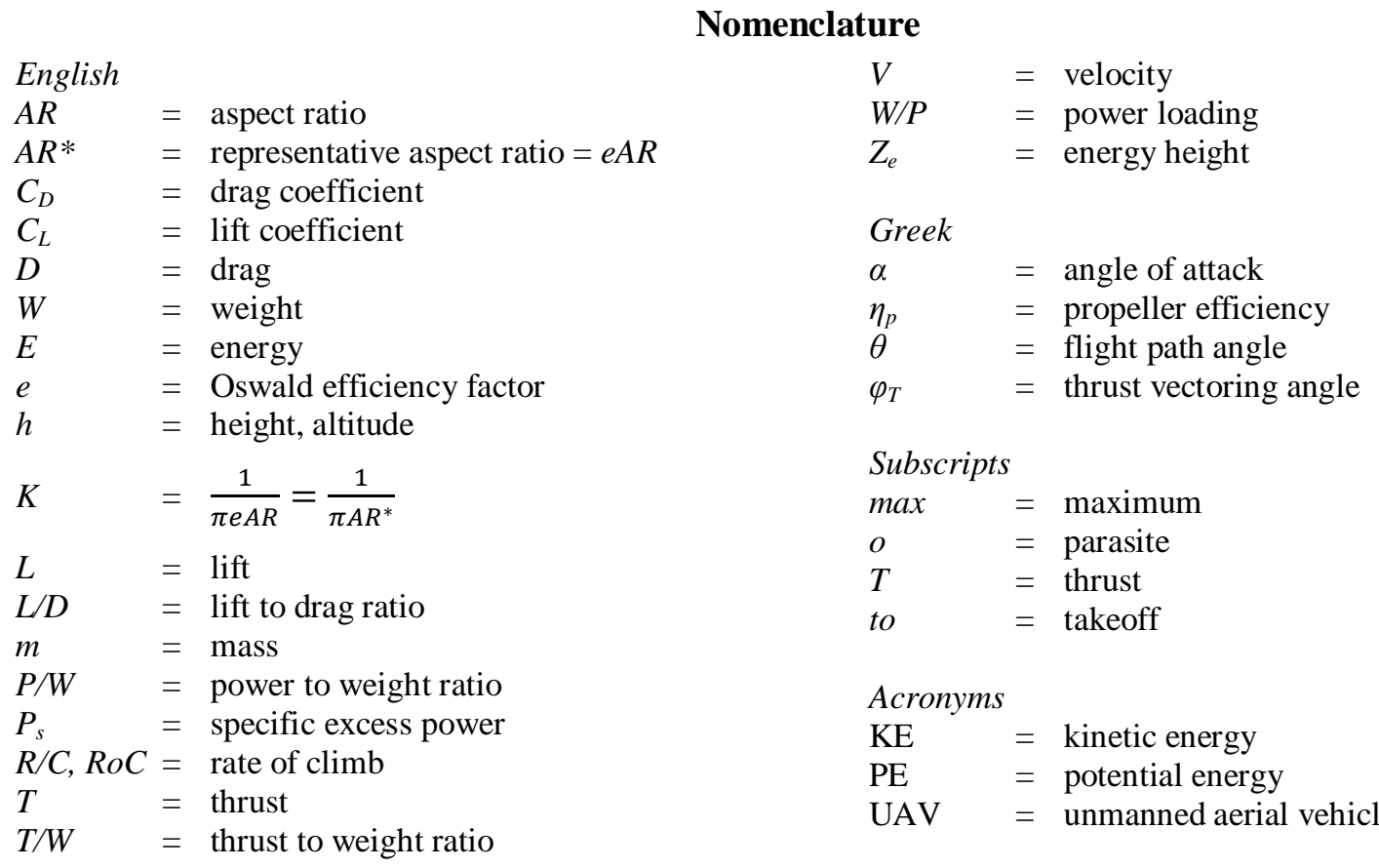
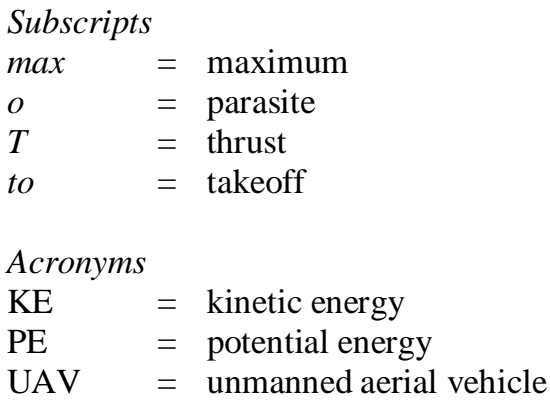

1Aerospace Engineering Undergraduate, AIAA Member 


\section{Introduction}

I

$\mathrm{T}$ is not typical for an aircraft designer to undersize the powerplant for their aircraft. However, as the emphasis on cost in the aerospace industry continues to increase, the need to meet customer requirements through the most cost effective means presents itself in almost all engineering problems. In a time where the cost of fuel is fluctuating unpredictably, manufacturing and labor costs are increasing, and high technology systems bring the development cost up, the need to perform and fly a mission must be as cost effective as possible. Using the technology of an "underpowered" aircraft (an aircraft that only has enough power to overcome some of the drag forces associated with flight) has the potential to reduce the operating and propulsion system cost when compared to standard aircraft systems that are capable of climbing or level flight. Possible mission applications for the technology include cargo delivery for deployed troops in the battlefield, glide munitions, stand-off weapons, and others.

The underpowered aircraft concept comes from the idea that, for specific missions, an aircraft system may not necessarily need to overcome all of the drag forces associated with flight, and can therefore operate with an undersized powerplant. If the purpose of the aircraft is to glide to its target, then the effective range of the aircraft can be increased by making the aircraft underpowered. By doing so, the aircraft does not need the same power as a standard aircraft would to achieve level or climbing flight, but can successfully operate off of smaller amounts of power while greatly extending the range of the vehicle. Adding power to the aircraft, in small amounts, allows the aircraft to increase the effective mission range, for little horsepower and low cost, as will be shown in this paper. The performance of an underpowered aircraft will be analyzed and the cost advantages will be determined. A more detailed numerical integration analysis of the flight path will also be presented and validation of the analysis will be conducted when compared to a glide munition operating off of the same flight principles.

\section{Underpowered Aircraft Performance}

The idea of an underpowered aircraft arises to fill the need to increase the range of gliding aircraft systems. Payload delivered using a gliding system provides for a cheap and simple delivery mechanism when constructed from light weight, inexpensive materials. The system can be designed to be single use or reusable, depending on the exact purpose. However, just using a standard gliding aircraft does not provide the range capability often needed for payload delivery. Assuming the underpowered aircraft is dropped from a high altitude, an engine can be sized and selected to provide the desired range. The trade space between range, flight velocity, and drop altitude for an underpowered aircraft will be explored. The following analysis will assume a payload delivery mission and then other mission profiles will be analyzed afterwards.

Two methods were used to determine the performance for the underpowered aircraft. First, the standard method of looking at the forces acting on the aircraft via a free-body diagram was applied. Estimates for the drag polar of the aircraft were also developed, which yielded an estimate of flight conditions. This was followed by a numerical integration to provide a more accurate measure and to account for thrust lapse. Emphasis was placed on the lift to drag ratio $(L / D)$ due to the vehicle's operation in gliding flight. In an abstract trade study such as this, aircraft characteristics are treated as technology that can be applied to an aircraft in the design process, allowing for an overall performance trade study.

\section{A. Equations of Motion Analysis}

To start, Figure 1 shows the standard forces acting on an aircraft in flight for use in the equations of motions analysis.

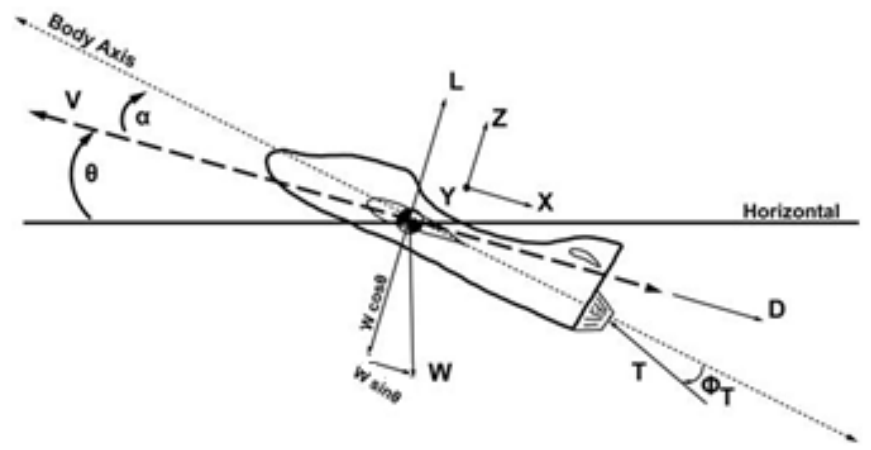

Figure 1. Forces Acting on an Aircraft in Flight

American Institute of Aeronautics and Astronautics 
The forces acting on the aircraft are standard and include the lift, drag, thrust, and weight of the aircraft. The velocity, flight path angle, $\theta$, and the angle of attack, $\alpha$, are also shown. The flight path angle is defined as the angle between the horizontal and the velocity vector and the angle of attack is defined as the angle between the velocity vector and the arbitrarily defined body axes for the aircraft. In the case of thrust vectoring, the thrust vector is offset by the angle $\varphi_{T}$, to model any effects from thrust vectoring on the performance of the aircraft.

This analysis assumes that there is no thrust vectoring happening in the flight of the aircraft. Thus, summing the forces in the $\mathrm{X}$ and $\mathrm{Z}$-axis for steady flight yields

$$
\begin{aligned}
& T=D+W \sin \theta \\
& L=W \cos \theta
\end{aligned}
$$

Thus, we reorganize equation 1 to find the classical aircraft performance equation

$$
\sin \theta=\frac{T-D}{W}
$$

For gliding flight, our interest lies in the flight path angle, $\theta$, of the vehicle. By minimizing the flight path angle to a small negative number, the range of the aircraft can be maximized. So, we have

$$
\theta=\sin ^{-1}\left(\frac{T}{W}-\frac{D}{W}\right)
$$

From equation 2 above and a small angle approximation, we have

$$
\theta=\sin ^{-1}\left(\frac{T}{W}-\frac{1}{\frac{L}{D}}\right)
$$

With the power to weight ratio (in units of hp/lb) defined as

$$
\frac{P}{W}=\left(\frac{T}{W}\right) \frac{V}{550 \eta_{p}}
$$

\section{B. Drag Polar}

One of the key characteristics needed to properly model the performance of an aircraft is an accurate representation of the drag polar for the entire aircraft. The form of the drag polar that will be used for the analysis is

$$
C_{D}=C_{D o}+\frac{C_{L}^{2}}{\pi A R^{*}}
$$

For the purposes of this study, the aspect ratio, $A R$, of the aircraft will be grouped together with the Oswald efficiency factor, $e$, to become a representative aspect ratio, $A R^{*}$. In an abstract trade study such as this no information is available on the efficiency of the wing design; however a feel for the efficiency factor can still be gained through a representative aspect ratio. If we assume that the aircraft is operating at conditions that give the $C_{L}$ for best $L / D$ for the gliding condition, then we know that ${ }^{1}$

$$
\begin{aligned}
C_{L}^{*} & =\sqrt{\frac{C_{D o}}{\pi A R^{*}}} \\
C_{D}^{*} & =2 C_{D o}
\end{aligned}
$$

So, the lift to drag ratio is

$$
\frac{L}{D}_{\max }=\frac{C_{L}}{C_{D}}=\frac{\sqrt{\frac{C_{D o}}{\pi A R^{*}}}}{2 C_{D o}}=\sqrt{\frac{1}{4 \pi A R^{*} C_{D o}}}
$$


Reorganizing, an estimate of the parasite drag can be determined through

$$
C_{D o}=\frac{\pi A R^{*}}{4\left(\frac{L}{D_{\max }}\right)^{2}}
$$

This allows us to treat the aerodynamics of the aircraft, $\mathrm{AR}^{*}$ and $\frac{L}{D_{\max }}$, as technology.

\section{Powerplant Cost Estimation}

With the performance for the underpowered aircraft concept established, the cost benefits of the technology were determined. Such a vehicle can be powered either by traditional internal combustion piston engines or small jet turbine engines. In order to get an estimate for the cost saving of the vehicle, estimates of small piston and jet turbine engines were developed. As seen below in Figure 2, a span of cost competitive small piston engines was used to determine a cost trend that was acquired through a survey of retail prices found online in August, 2008. These engines included small RC aircraft engines from O.S. Engines, general purpose engines from Honda and Kawasaki, as well as a few larger Rotax aircraft engines. The model yields an excellent correlation coefficient and the results are consistent with the investigative nature of this paper.

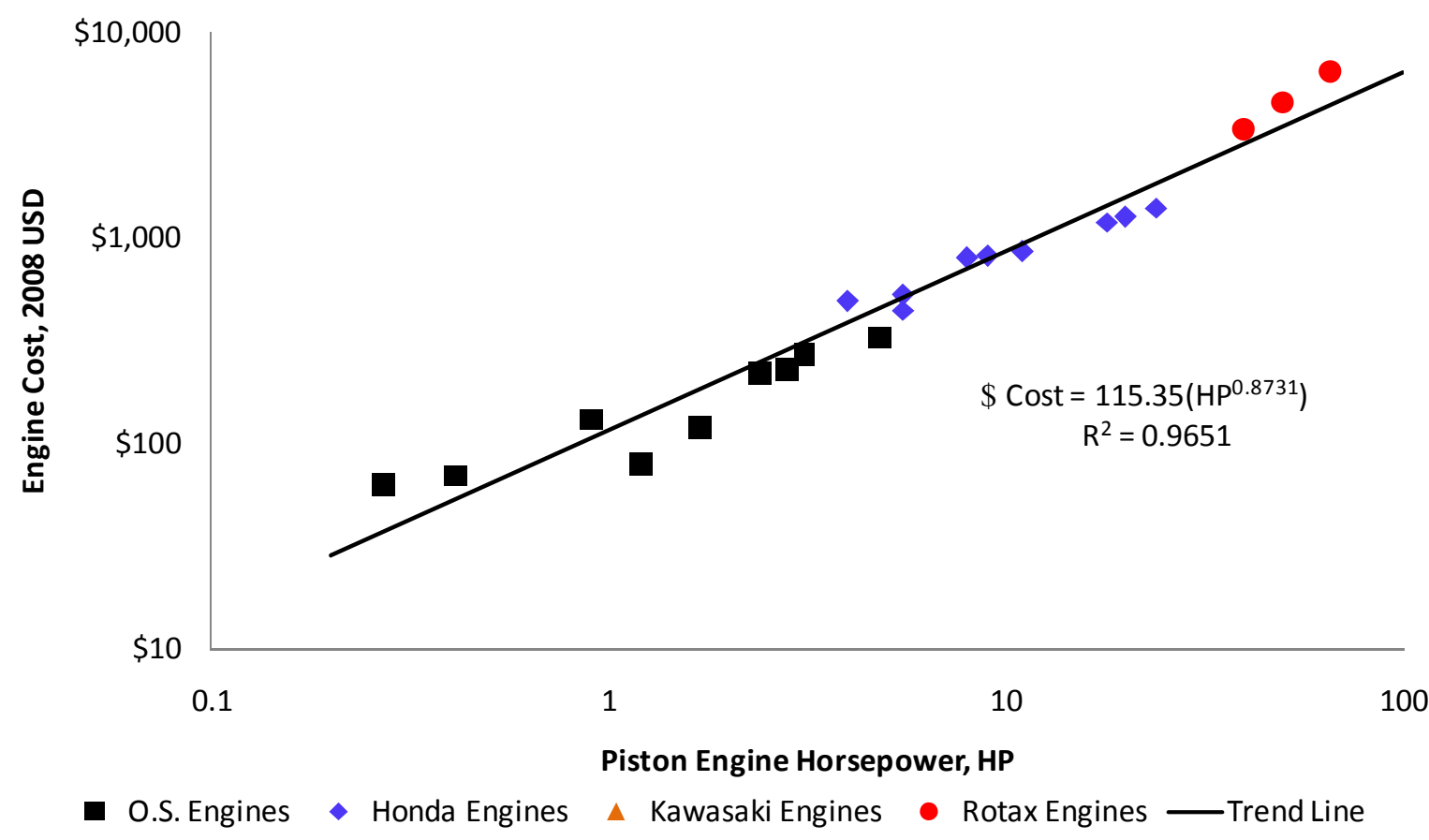

Figure 2. Small Piston Engine Cost

Figure 3 shows a similar trend for small jet turbine engines. Due to the fact that the payload delivery vehicles and stand-off "glide munitions" where this technology would be used are not large in size, the engine study was limited to model aircraft jet engines as well as jet engines used in small UAV applications. These engines include manufacturers such as JetCat, foreign engine manufacturers such as SimJet, and others. This model is indicative of the low thrust engines needed for underpowered applications. The model yields acceptable results with a correlation coefficient of $82 \%$. 


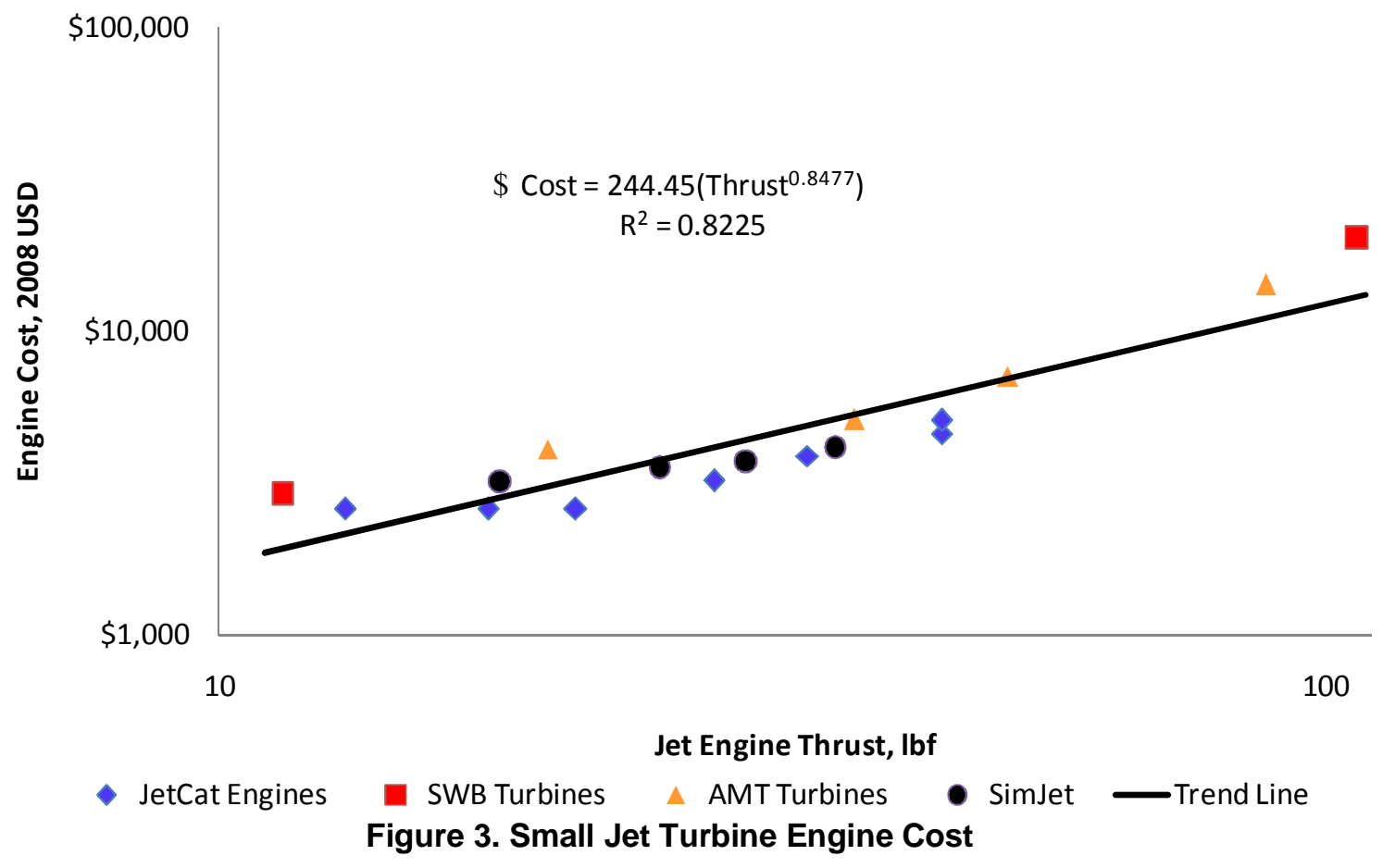

\section{Underpowered Aircraft Flight Performance}

Since the concept for this aircraft is based on that of a glider, the performance of the aircraft has been determined to represent the trade space of velocity, range, and drop altitude. So, using the relationships derived earlier and performance code written in MATLAB, some performance trends were calculated.

It is assumed in this analysis that the underpowered aircraft is dropped from altitude at the start of the flight. Two altitudes of 10,000 feet and 25,000 feet were chosen to give a comparison between a low altitude drop and a high altitude drop. The drop aircraft could consist of any type of vehicle that is capable of carrying the underpowered aircraft to the drop altitude. Winds aloft are not included in the analysis. The analysis presented assumes that the underpowered vehicle is dropped at the velocity of best $L / D$ and that during the glide the vehicle operates at the best $L / D$ for glide.

As the lift to drag ratio is increased, the thrust required to get the aircraft to reach its destination decreases, which can be seen in Figure 4. Only a small amount of thrust is required to keep the aircraft in a level flight condition, but a significantly larger amount of thrust is needed to have a positive rate of climb for the aircraft (represented by the +3 degree climb angle curve). The difference in gliding flight, level flight, and positive rate of climb flight is indicative of the trade space between range, velocity, and drop altitude (10,000 feet for these curves). It is also indicative of the trade in the cost of the system, as having an aircraft that is capable of positive rate of climb requires a significantly larger amount of thrust and therefore a larger, more expensive powerplant. It is important to note that there are no additional assumptions built into the figure below and the plot is derived solely from aircraft performance metrics. 


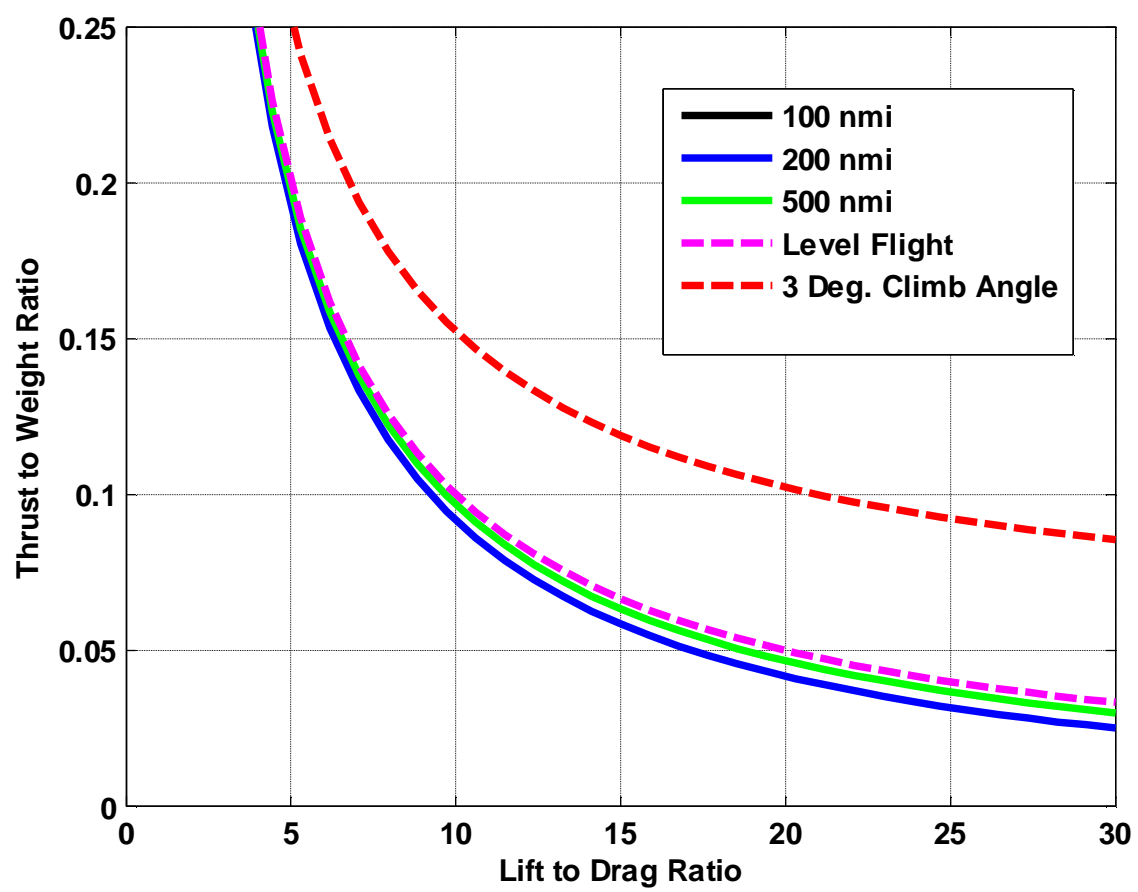

Figure 4. Underpowered Jet Aircraft Performance from a 10,000 ft Drop Altitude

The curves in Fig. 4 are a function of thrust to weight ratio and lift to drag ratio which are two of the most fundamental aircraft performance parameters. For these initial calculations the thrust lapse has been ignored. Using the cost model presented earlier we can estimate the cost saving of the underpowered aircraft technology. Assuming that the vehicle has a takeoff gross weight (TOGW) of 1,500 pounds and can achieve a lift-to-drag ratio $(L / D)$ of 20 , the vehicle requires a thrust to weight ratio 0.04 to achieve a range of $200 \mathrm{nmi}$. This corresponds to a thrust required of $60 \mathrm{lbf}$ and an engine cost of $\$ 7,862$. In contrast, the engine would need to provide $75 \mathrm{lbf}$ for level flight and 150 lbf for climbing flight. This yields a cost of $\$ 9,500$ and $\$ 17,095$, respectively.

The same curves can be represented for a propeller driven aircraft by including flight velocity and propeller efficiency. Shown in Figure 5 are the same trends as a function of power to weight ratio.

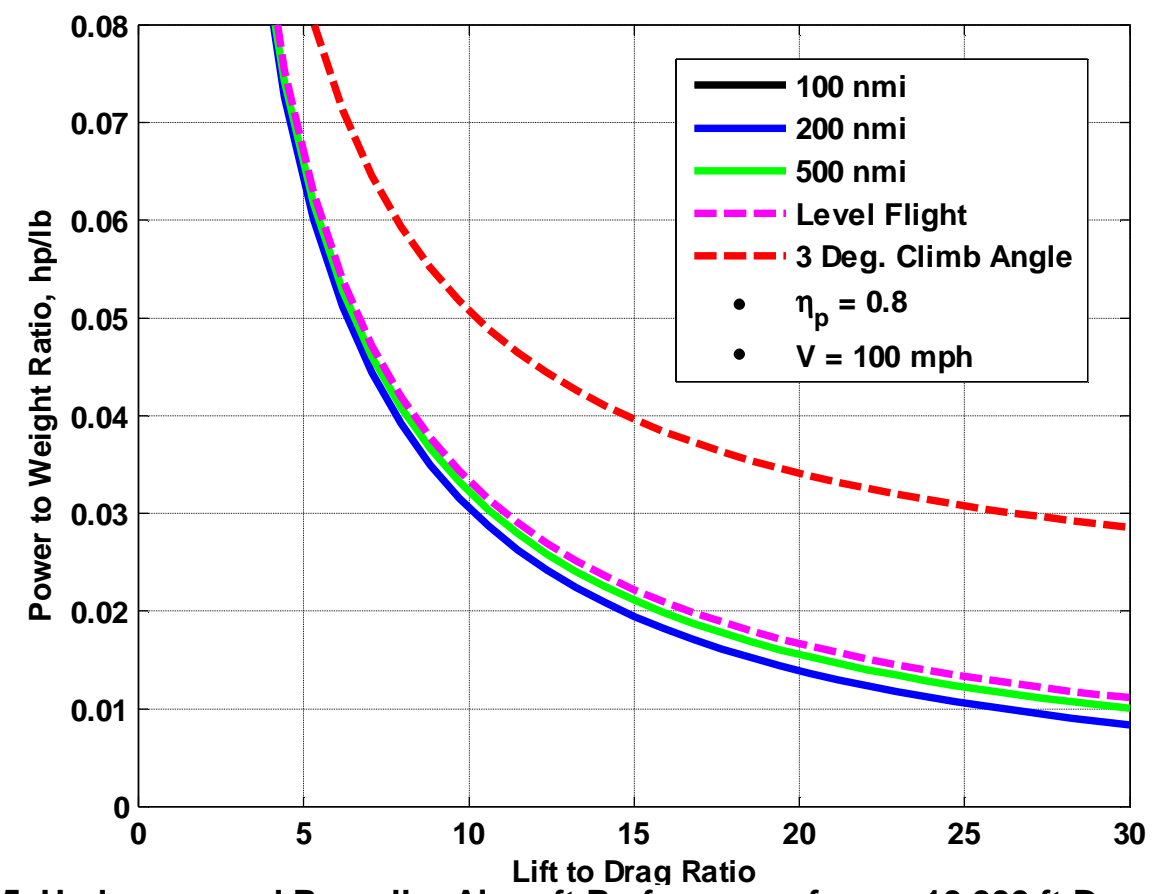

Figure 5. Underpowered Propeller Aircraft Performance from a 10,000 ft Drop Altitude

American Institute of Aeronautics and Astronautics 
Figure 5 shows similar trends to Figure 4 . This plot speaks volumes about the underpowered aircraft concept. If we assume that the underpowered aircraft can achieve a maximum lift to drag ratio of 20 and has a TOGW of 1,500 pounds, then the aircraft only requires about $20 \mathrm{hp}$ to achieve a range of $200 \mathrm{nmi}$ (even less for shorter ranges). The same aircraft would require $26 \mathrm{hp}$ for level flight, and about $53 \mathrm{hp}$ for climbing flight. The underpowered aircraft requires over one half the horsepower to achieve the mission requirement which translates to engine costs of $\$ 1,577$, $\$ 1,983$, and $\$ 3,694$ respectively. There are two main assumptions built into this analysis: First, a propeller efficiency of 0.80 is assumed to convert from thrust to horsepower ( $80 \%$ being typical performance of most propellers currently used today) and second, an operating velocity of $100 \mathrm{mph}(146.6 \mathrm{ft} / \mathrm{sec})$ was arbitrarily chosen to represent a reasonable delivery speed for the payload aircraft.

A different presentation of the data in Fig. 5, seen below in Fig. 6, shows the relationship between power loading and lift to drag ratio for the underpowered aircraft.

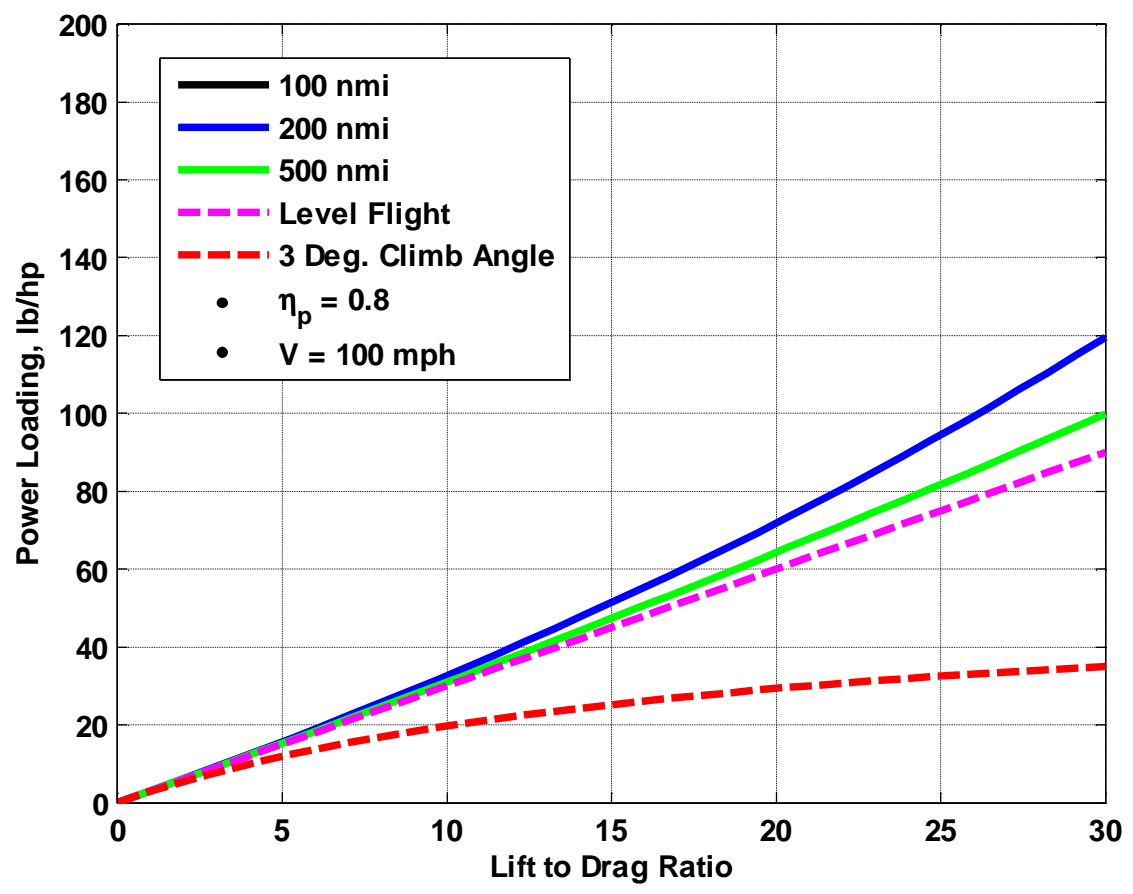

Figure 6. Underpowered Aircraft Power Loading Requirements for a 10,000 ft Drop Altitude

This figure shows how much of the aircraft (in weight) can be supported for each unit of horsepower that the engine on the aircraft will produce. If we again assume that the aircraft has a lift to drag ratio of 20 and a weight of 1,500 pounds, then the aircraft can carry about 76 pounds for each horsepower. This results in a power to weight ratio of $0.013 \mathrm{hp} / \mathrm{lb}$ and about a $20 \mathrm{hp}$ engine as shown above in the previous figures. This analysis also assumes a propeller efficiency of 0.80 and a flight velocity of $100 \mathrm{mph}$ as mentioned earlier.

The same graphs were created to look at the performance of the aircraft from the drop altitude of 25,000 feet. Note the significantly less power required for the underpowered aircraft to achieve a desired range of $200 \mathrm{nmi}$ in Figure 7. 


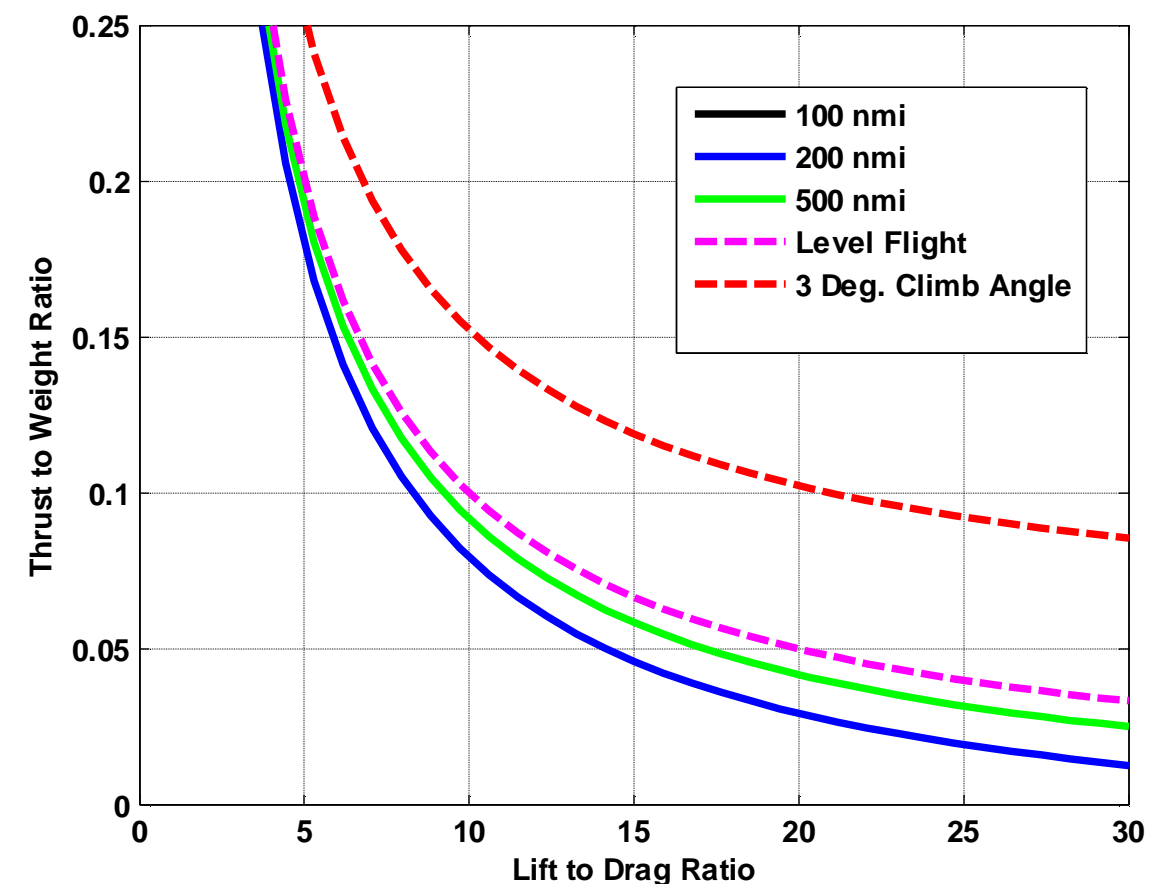

Figure 7. Underpowered Jet Aircraft Glide Performance from a 25,000 ft Drop Altitude

If we continue to assume that the aircraft has a TOGW of 1,500 pounds and can achieve a lift-to-drag ratio of 20, the vehicle will require a thrust to weight ratio of 0.03 to achieve a $200 \mathrm{nmi}$ range, a thrust to weight ratio of 0.05 to achieve level flight, and a thrust to weight ratio of 0.1 to achieve a positive rate of climb. From the cost models presented above, the engine would need $45 \mathrm{lbf}$ thrust, $75 \mathrm{lbf}$ thrust, and $150 \mathrm{lbf}$ thrust respectively. This would represent a significant cost savings between $\$ 6,161, \$ 9,500$, and $\$ 17,095$ respectively.

Shown in Figure 8 is the relationship between power to weight ratio and lift to drag ratio.

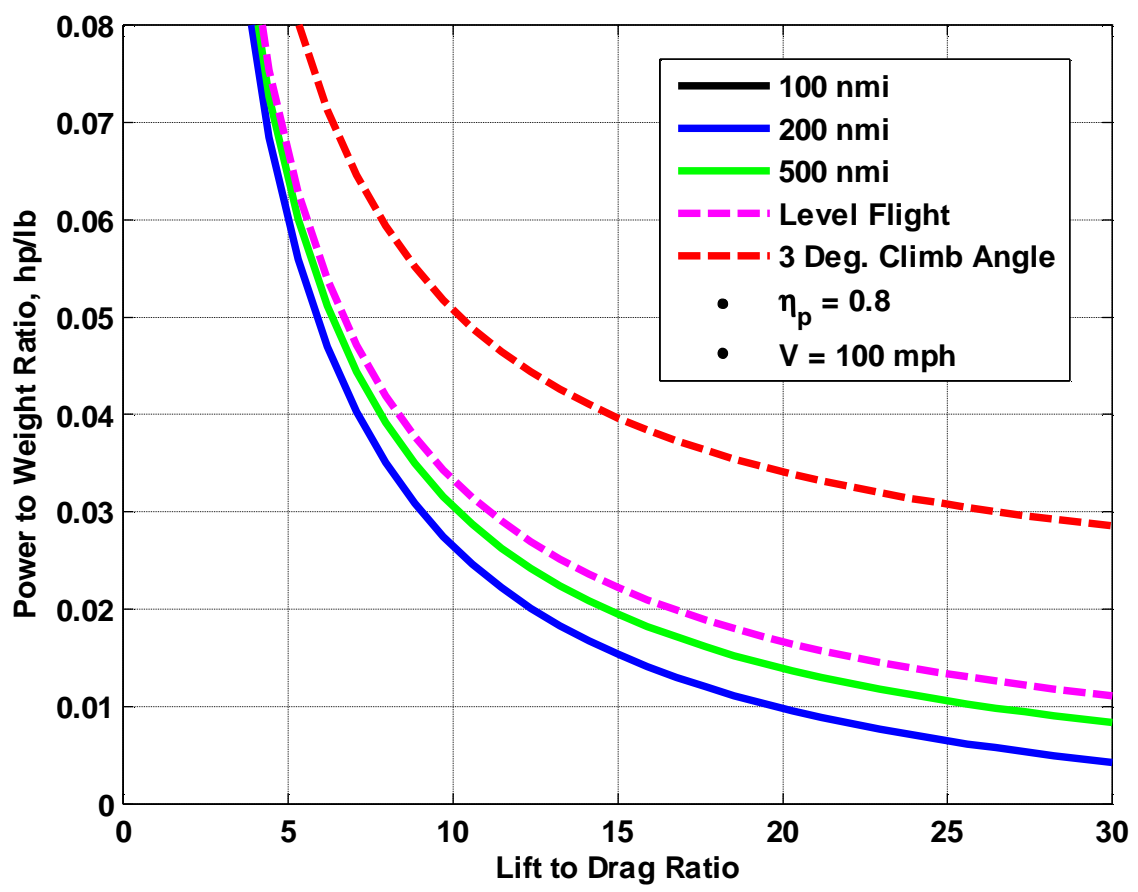

Figure 8. Underpowered Propeller Aircraft Performance from a 25,000 ft Drop Altitude

American Institute of Aeronautics and Astronautics 
Using the same assumptions as before and looking at an aircraft that can achieve an aerodynamic lift to drag ratio of 20 , and has a weight of 1,500 pounds, the aircraft will require a power to weight ratio of $0.01 \mathrm{hp} / \mathrm{lb}$ or $15 \mathrm{hp}$ to reach the desired range of $200 \mathrm{nmi}$. This would be a minimal cost of $\$ 1,227$. This is significantly less than an aircraft that would need to sustain level flight (approx. $26 \mathrm{hp}$ or $\$ 1,983$ ) and an aircraft looking to have a positive rate of climb (approx. $53 \mathrm{hp}$ or \$3,694). The underpowered aircraft technology will satisfy the requirements with significantly less cost due to the much smaller engine required which reduces cost throughout the life cycle. These values are sensitive to wing technology (lift to drag ratio) and will change depending on the $L / D$ that can be achieved for the vehicle.

The power loading for the underpowered aircraft also significantly changes for the additional drop altitude of 25,000 feet and allows the aircraft to glide a longer distance with less power.

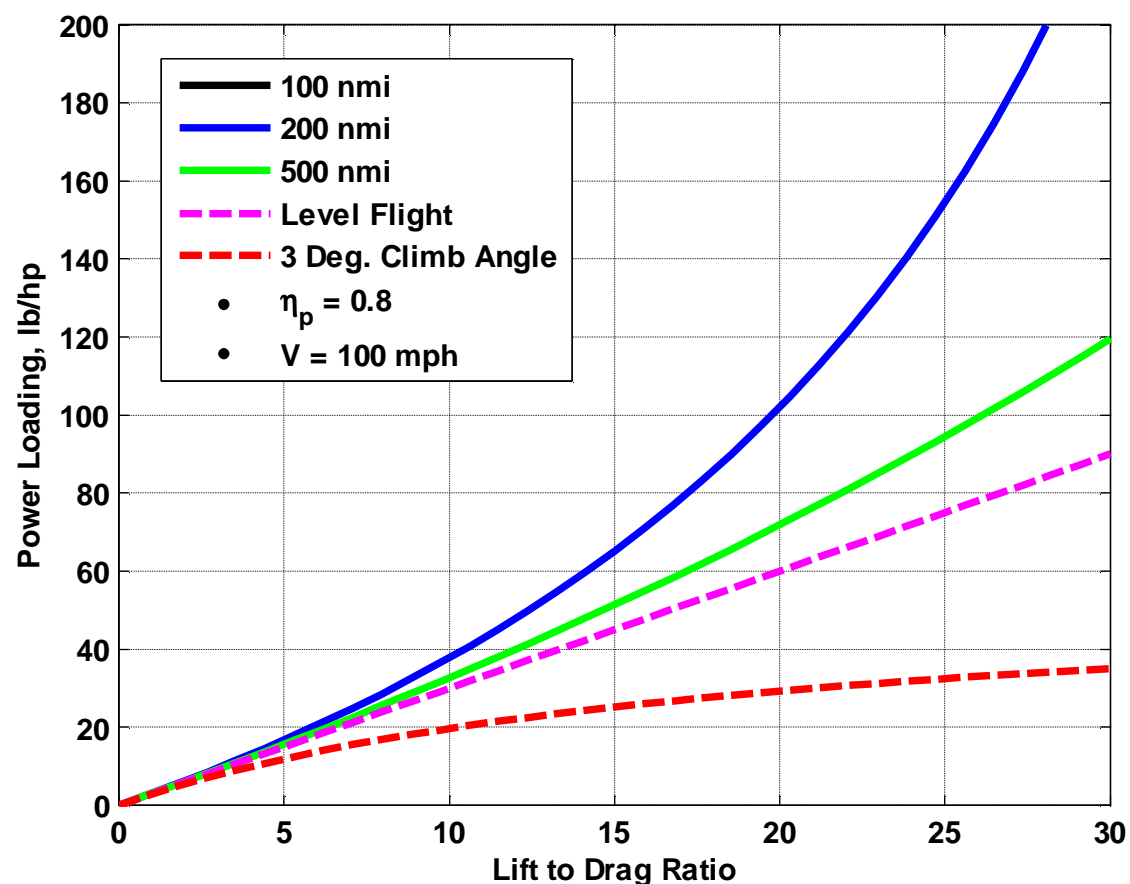

Figure 9. Underpowered Aircraft Power Loading Requirements for a 25,000 ft Drop Altitude

Comparing the two drop altitudes shows significant savings as well. Using the same aircraft assumptions as before, namely a TOGW of 1,500 pounds and a maximum lift to drag ratio of 20 , we see that a drop from 10,000 ft achieves $200 \mathrm{nmi}$ with a $60 \mathrm{lbf}$ engine and an engine cost of $\$ 7,862$. This cost is decreased when dropped from $25,000 \mathrm{ft}$, when the plane achieves the same range with a $45 \mathrm{lbf}$ engine and an engine cost of $\$ 6,161$.

\section{Numerical Integration Approach}

While the above analysis represents a "back-of-the-envelope approach", there is one main factor that was left out; the effects of velocity and altitude on powerplant performance. This takes the form of a thrust lapse that will change the maximum thrust to weight ratio represented in the earlier figures, to a typical flight thrust to weight ratio that has been adjusted to represent actual flight conditions. To achieve this, a numerical integration approach was used to examine the equations of motion, take a thrust lapse into account, and give more accurate results than the methods presented above.

Revisiting the free body diagram in Figure 1 and summing the forces in the $\mathrm{X}$ and Z-axis, we have

$$
\begin{aligned}
& \cos \theta=\frac{L}{W} \\
& \sin \theta=\frac{T-D}{W}
\end{aligned}
$$

American Institute of Aeronautics and Astronautics 
Due to the fact that our interest lies in gliding flight, we are actually interested in the change in altitude of the vehicle during flight with respect to the change in distance the vehicle travels along the ground. This is essentially the glide ratio in derivative form. So, using the free body diagram

$$
\begin{aligned}
& \frac{d s}{d h}=\frac{\frac{d s}{d t}}{\frac{d h}{d t}}=\frac{V \cos \theta}{V \sin \theta}=\frac{1}{\tan \theta} \\
& \frac{1}{\tan \theta}=\frac{L}{T-D}
\end{aligned}
$$

Using a thrust lapse equation from Mattingly ${ }^{2}$ defined in terms of the Mach number, $M$, and the density ratio from sea level, $\sigma$, we have

$$
\alpha=0.76\left\{0.907+0.262(|M-0.5|)^{1.5}\right\} \sigma^{0.7}
$$

Setting up the integral to integrate the flight path during steady flight, we have

$$
\begin{aligned}
& \int_{h}^{0}\left(\frac{\frac{1}{W}}{\frac{1}{W}}\right) \frac{L}{\alpha T-D} d h \\
& \int_{h}^{0}\left(\frac{1}{\alpha \frac{T}{W}-\frac{1}{\frac{L}{D}}}\right) d h
\end{aligned}
$$

The numerical integration yielded results that provide a better representation of the flight regime with the inclusion of a thrust lapse. The trend in the curves shows that the numerical model actually requires more thrust than the equation of motion or energy method analysis because it now takes powerplant performance into account. The powerplant will not perform at sea level static conditions at high altitude. In an effort to validate the results of the model presented above, a drop altitude of 40,000 ft was used to match the ranges given for the AGM-154 Joint Standoff Weapon ${ }^{3}$. Figure 10 shows the results of the numerical integration.

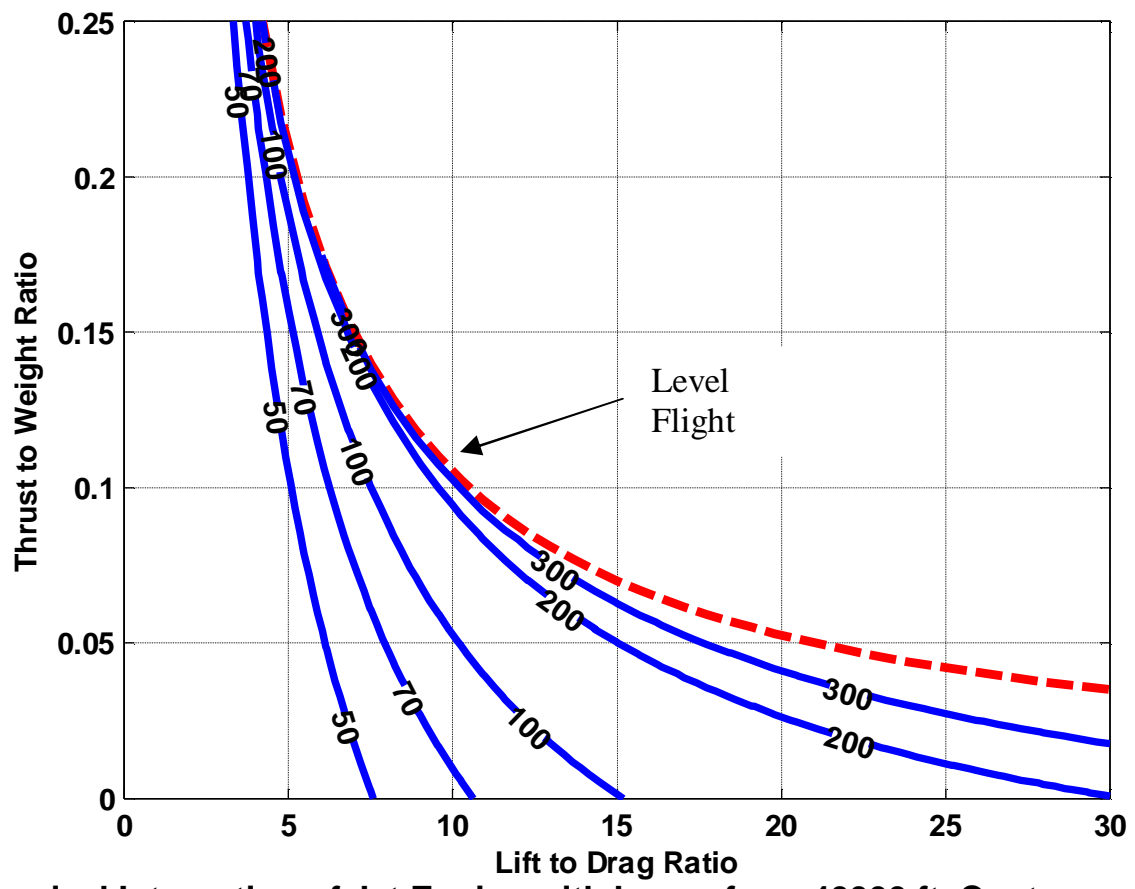

Figure 10. Numerical Integration of Jet Engine with Lapse from $40000 \mathrm{ft}$, Contours of Glide Range

American Institute of Aeronautics and Astronautics 
According to the AGM-154 product card ${ }^{3}$ the unpowered version of the JSOW has an effective glide range of 70 nmi from a 40,000 ft drop altitude. When using the analysis of Figure 10, for an un-powered vehicle, this corresponds to a lift to drag ratio of about 11. The weight reported for this vehicle is 1,050 lbf (depending on variant $)^{3}$. Recently reported in a Raytheon press release ${ }^{4}$ was a powered version of the JSOW, the JSOW-ER, with a $300 \mathrm{nmi}$ range using a $150 \mathrm{lbf}$ thrust turbojet engine. If we assume that the powered version is also dropped from $40,000 \mathrm{ft}$, and using the same $L / D$ as determined for the un-powered version, the weight of the powered JSOW-ER was determined to be 1,575 lbf. This is very close to the weight reported for the un-powered version, and shows that the JSOW-ER is most likely an "underpowered" vehicle, utilizing the technology described in this paper.

As an estimation technique, the simpler equation of motion analysis was calibrated to match the results of the numerical integration analysis. Figure 11 shows the same curves for the simpler analysis, including the same thrust lapse model presented above, but the density ratio is estimated at an altitude of 18,000 $\mathrm{ft}$. This was considered a good estimate for our purposes of modeling the flight regime because most of the effects of the thrust lapse are seen at lower altitudes for longer ranges.

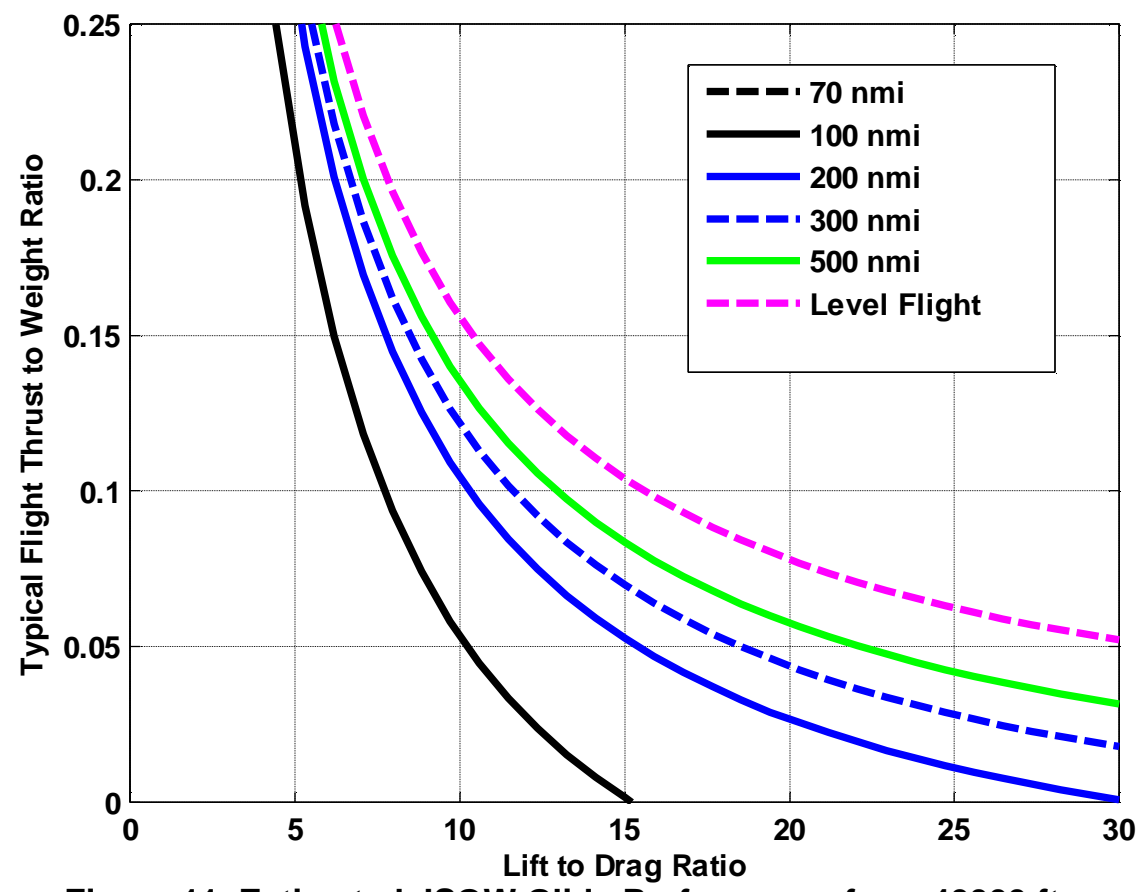

Figure 11. Estimated JSOW Glide Performance from $40000 \mathrm{ft}$

\section{Conclusion}

An underpowered aircraft concept was studied to determine the feasibility and cost effectiveness of such a technology, as well as possible applications. It was determined, through an equations of motion, energy method, and numerical integration analysis, that an underpowered aircraft can provide significant range extension for a gliding flight vehicle. The methods also include a built in thrust lapse to correctly model an underpowered vehicle's performance with altitude. Also, the methods developed in this paper were compared with a current gliding vehicle, the AGM-154 JSOW and JSOW-ER. It was determined, from aircraft metrics provided by Raytheon and our analysis, that the JSOW-ER is most likely an underpowered aircraft and is representative of a possible mission application for the technology. Other applications include payload delivery, glide munitions, UAVs, and others. Overall, the underpowered aircraft technology represents a unique flight regime, giving great benefit to the user for a low overall cost. 


\section{References}

${ }^{1}$ Anderson, John D., Aircraft Performance and Design, WCB/McGraw-Hill, 1998

${ }^{2}$ Mattingly, J. D., Heiser, W. H., and Daley, D. H., Aircraft Engine Design, AIAA Education Series, AIAA, New York, 1987, Chap. 2.

${ }^{3}$ Raytheon Company. JSOW: Family of Precision Strike Weapons. Brochure. Tucson: Raytheon Company, Missile Systems, 2008.

${ }^{4}$ Raytheon. Press release. Raytheon Demonstrates Engine for Powered Joint Standoff Weapon Extended Range. 20 Feb. 2007. Aug. 2008 <http://www.globalsecurity.org/military/library/news/2007/02/mil-070220raytheon $02 . \mathrm{htm}>$. 